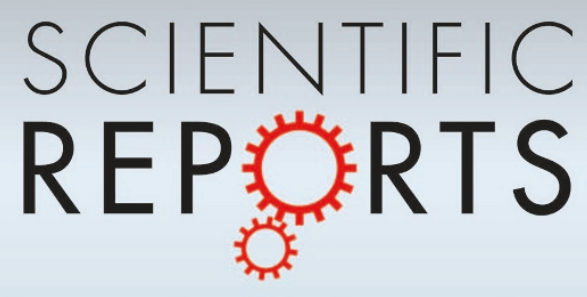

OPEN

SUBJECT AREAS:

SENSORS AND

BIOSENSORS

BIOMATERIALS

BIOMEDICAL ENGINEERING

Received

27 December 2013

Accepted

17 February 2014

Published

6 March 2014

Correspondence and requests for materials should be addressed to F.G. (gentile@unicz.it)

\section{Diffusion Driven Selectivity in Organic Electrochemical Transistors}

\author{
Nicola Coppedè' , Marco Villani' \& Francesco Gentilee,3 \\ ${ }^{1}$ IMEM-CNR Parco Area delle Scienze 37/A - 43124 Parma, Italy, ${ }^{2}$ Istituto Italiano di Tecnologia, Via Morego 30 - 16163 Genova, \\ Italy, ${ }^{3}$ BioNEM, University Magna Graecia of Catanzaro - 88100 , Italy.
}

\begin{abstract}
Organic Electrochemical transistors (OECTs) present unique features for their strategic combination with biomedical interfaces, simple and low voltage operation regime and sensing ability in aqueous environment, but they still lack selectivity, so that a significant effort in research is devoted to overcome this limitation. Here, we focus on the diffusion properties of molecular species in the electrolyte, which opportunely analyzed, modeled and compared to experimental data, serve as a simple and direct key factor in the recognition of species during OECT sensing. Specifically, we model the transient behavior of an OECT considering the effect of diffusion of the target species in the electrolyte. In doing so, we develop a general method that can be used to differentiate and distinguish different molecules from a complex mixture, on the basis of their diffusivity and thus mass. More importantly, the model can be realistically used to determine the physical characteristics of the transported species in a solution from a simple fitting procedure. On the basis of the obtained results, we discuss the contribution that our study could bring to OECT architecture to realize a new generation of devices with improved sensitivity, selectivity and reliability.
\end{abstract}

ince the discovery of conducting polymers in the mid-1970s ${ }^{1}$, the development of innovative devices based on their structural and conducting properties has grown interest for application in organic electronics and bioelectronics. Organic Field effect Transistors based on different organic structure have been realized, with promising application in printed and flexible electronics ${ }^{2}$. Among the different approaches to face organic electronic challenges, much attention has been dedicated to devices which exploit the interactions of organic polymer with ionic electrolytes: Organic Electrochemical transistors (OECTs) present unique features for their strategic combination with biomedical interfaces ${ }^{3}$, simple and low voltage operation regime ${ }^{4}$ and their sensing ability in aqueous environment ${ }^{5}$. OECT bases its working principles on the de-doping action of a liquid or gel electrolyte over the conducting channel of a polymeric film. The interaction, between ionic species in the electrolyte and the active area of the organic conductive polymer, controls the modulation process in the transistor, used for either electronic or sensing applications $s^{6,7}$. One of the most used p-type organic semiconductor is Poly(3,4-ethylenedioxythiophene) doped with poly(styrenesulfonate) (PEDOT:PSS), which presents high stability in a wide $\mathrm{pH}$ range, high conductivity, low cost and lithographic process integration ${ }^{8}$. OECT devices based on PEDOT:PSS have been employed in different technological fields such as chemical and biological sensing ${ }^{9}$, control of cell adhesion ${ }^{10}$ and viability ${ }^{11}$, sport and healthcare monitoring through integration on natural cotton fiber ${ }^{12}$. Among the different applications, one of the crucial challenges to realize effective identification of the ionic species in-sensing and bio-sensing is to develop and improve electivity properties. The ability to reveal with high sensitivity different species in electrolyte solutions has been demonstrated ${ }^{7,13}$, nevertheless recognizing the different species revealed is the actual challenge to be faced, and this requires different strategies ${ }^{14,15}$. Among the possible approaches, one of the most simple and direct is to evaluate the different diffusion properties of the ionic species in the electrolyte. In considering the physical characteristics which influence the diffusion properties of the ionic species it is possible, using a correct model for the OECT working processes, to distinguish different ionic species. Previously, an important model for charge transport in organic electrochemical transistor has been proposed by Bernards and al. ${ }^{16}$, which takes care of a complex interplay between ionic and electronic motion. In particular, a separation between ionic transport in the electrolyte and hole transport in the PEDOT:PSS channel is considered. The result is that the hole electronic charge is modeled as a semiconductor, while the ionic charge is considered on the direct effect of a drift voltage. Using an ionic circuit equivalent toideal polarizable electrodes, this model reproduces the steady stateand transient characteristics of PEDOT:PSS OECTs, but it disregards the effect of diffusion of the ionized species in the electrolyte.

Here, we revised the model of Bernards to consider the effectof diffusion. Using the formalism of the Nernst-Planck equations ${ }^{17-19}$ (Methods) we described the motion of the charged species in the electrolytein terms 
of the diffusivity and charge of those species (Figure 1). This motion, in turn, results crucial to determine the effective behavior of drain source current in dependence to the physical properties of ionic species.

In comparing experimental results of a real OECT device with the predictions of our theoretical model, we recognized and identified four different species (that are, $\mathrm{Na}, \mathrm{Mg}, \mathrm{K}, \mathrm{Zn}$ ) in a solution on the basis of their different mass and charge (and thus diffusivity). Moreover, from a simple fitting procedure, we estimated the numerical values for the diffusion coefficients of the released species with good accuracy.

The model effectively predicts the different kinetic behavior of the source-drain current for the different species, allowing a recognition and identifications of the ionic salts, improving the selectivity of the device. The results pave the way to an innovative kinetic analysis of the OECT modulations, useful for selective recognition of ionic species, with many sensing and bio-sensing applications.

\section{Results}

Effect of diffusion. On varying the diffusion coefficient over a significant range, we observed a very high sensitivity of the transient behavior of the OCET on the diffusivity of the ionized species in the electrolyte. Fig. 2a reproduces the simulated transient current $\mathrm{I}(\mathrm{t})$ as a function of time for the effective diffusion coefficient varying from $10^{-1}$ to 10 times $D_{o}$, where $D_{o}=10^{-8} \mathrm{~m}^{2} / \mathrm{s}$ is an intermediate value of diffusivity used as a reference. In the considered case, $z=1$ is hold fixed, while the remaining variables utilized in the simulations were adjusted as follows: $\varphi=1 \mathrm{~V}, \mathrm{x}=1 \mathrm{~cm}, \mathrm{E}_{\mathrm{x}}=-\partial \varphi / \partial \mathrm{x}=100 \mathrm{~V} / \mathrm{m}$, $\mathrm{T}=298 \mathrm{~K}, \mu=510^{26} \mathrm{~m}^{2} /(\mathrm{V} \mathrm{s}), \mathrm{p}_{\mathrm{o}}=510^{-4} \mathrm{~m}^{-3} \mathrm{eV}^{-1}, \mathrm{q}=10^{-19} \mathrm{C}$.

For fixed values of $\mathrm{D}$, the current increases for increasing time; however, the exact time behavior of the system would significantly depend on the magnitude of $\mathrm{D}$. For low values of $\mathrm{D}$, that is, for $\mathrm{D} / \mathrm{D}_{\mathrm{o}}$ $<1.5$, the sensor behaves as if the output conforms to the standard curve of a first-order response, where the rise time and the time constant, that is here defined as the time which is required by the sensor to reach $90 \%$ of the steady-state or maximum level upon application of the external voltage, diminish with D. For values of $\mathrm{D} / \mathrm{D}_{\mathrm{o}}$ larger than 1.5 , the time-dependent characteristic of the system resembles that of a slightly undamped second order system, here, the response is even faster with the typical overshoot of similar dynamic systems. Fig. $2 c$ reports the simulated current as a function of $\gamma=D /$ $D_{0}$ for defined values of time, the derivative $\partial \mathrm{I} / \partial \gamma$ of this diagram is reported separately in Fig. 2e; they both provide information about the sensitivity of the current to the diffusion coefficient. Collectively, these results indicate that the effect of diffusion is moderate at the early time of the process, while it is significant for larger values of time. Moreover, the sensitivity of the sensor would depend on D. For vanishingly small values of diffusion, that is, for $\gamma \rightarrow 0$, the intensity of current will vary significantly for small variations of D. At any time of the simulated process of measurement, the current is proportional to $\mathrm{D}$, and this is easily explained considering that augmenting $\mathrm{D}$ is equivalent to using smaller molecules, that can be more easily transported from the electrolyte to the PEDOT conductive polymer.

Effect of charge. In this section, the effect of charge on the overall response of the system is analyzed. In doing so, the diffusion coefficient is maintained constant as $\mathrm{D}=$ Do, while the charge number $\mathrm{z}$ is varied from 0.1 to 10 . The simulated transient current $\mathrm{I}(\mathrm{t})$ is reproduced in Fig. $2 \mathrm{~b}$ as a function of time for these prescribed values of $\mathrm{z}$. For a fixed $\mathrm{z}$, the current smoothly transitions from zero to a steady state value that increases for increasing $z$, and the sensor behaves like a first order system. Differently from the case of diffusion, the behavior of the system is similar for any charge number, and thus $\mathrm{z}$ may be considered like a simple scale factor. This is even more evident in Fig. 2d, where $\mathrm{I}$ is reported as an explicit function of $\mathrm{z}$ at different times. When $\mathrm{t}$ is small, that is, approximately for $t<8 \mathrm{~s}$, the current $\mathrm{I}$ is constant over $\mathrm{z}$. For large values of time, that is, $\mathrm{t}>8 \mathrm{~s}$, I is quasi linear in $\mathrm{z}$, and thus the effects of $z$ on I are easily predicted. However, these effects are small, in the sense that the variations of I are limited on changing $\mathrm{z}$ (Fig. 2d). The analysis of sensitivity to $z$ (Fig. 2f) confirms this understanding. Overall, on comparing Fig. $2 \mathrm{e}$ and Fig. $2 \mathrm{f}$ it appears that the contribution of diffusion on the absolute magnitude of ionic current dominates over the contribution of charge number. Or, that is equivalent, the system responds more promptly to variations in $\mathrm{D}$, compared to variations in the charge number $\mathrm{z}$.

Combined effect of diffusion and charge on the current settling and rise time. The model here introduced makes it possible to predict the current response of an OECT as a function of diffusion and charge. The concerted action of these two parameters and their influence on the time constant $\tau$ and rising time of the device is analyzed and reported in Figure 3.In Fig. 3a, the current settling time is shown in the form of a contour plot as a function of $\mathrm{D} / \mathrm{D}_{\mathrm{o}}$ and $\mathrm{z} / \mathrm{z}_{\mathrm{o}}\left(\mathrm{D}_{\mathrm{o}}=10^{-8} \mathrm{~m}^{2} / \mathrm{s}, \mathrm{z}_{\mathrm{o}}=1\right)$. The time to the steady state behavior of the device is large for small values of the diffusion coefficient, and gradually decreases for increasing D. Notice, from the diagram, that $\mathrm{D}$ dominates over $\mathrm{z}$. For vanishingly small values of $\mathrm{D}$ to nearly $\mathrm{D}=4 \mathrm{D}_{\mathrm{o}}$, the influence of $\mathrm{z}$ can be neglected, and the time constant does not vary on changing $z$. This scenario changes for larger values of $\mathrm{D}$. On augmenting $\mathrm{D}$ or, equivalently, on considering smaller molecules, the effect of the charge cannot be disregarded. Low values of $t$ are thus obtained in the region of the diagram where $\mathrm{D}$ and $\mathrm{z}$ are simultaneously large. For the particular set of values considered here, the time constant of the systems varies from $\sim 60 \mathrm{~s}$, corresponding to diffusion coefficients smaller or equal than about $D_{o}$, to less than $13 \mathrm{~s}$, that instead is obtained for $\mathrm{D}$ and $\mathrm{z}$ larger than, respectively, 8 Do and $3 \mathrm{z}_{\mathrm{o}}$.

An important parameter for characterizing the time response of the system, is the rising time $\mathrm{k}$, that is here defined as the time derivative of the ionic current calculated at the early time of the process (that is, $t=0$ ). It gives a measure of how rapidly the current increases. In Fig. $3 \mathrm{~b}, \mathrm{k}$ is reported as a function of $\mathrm{D}$ and $\mathrm{z}$. Similarly

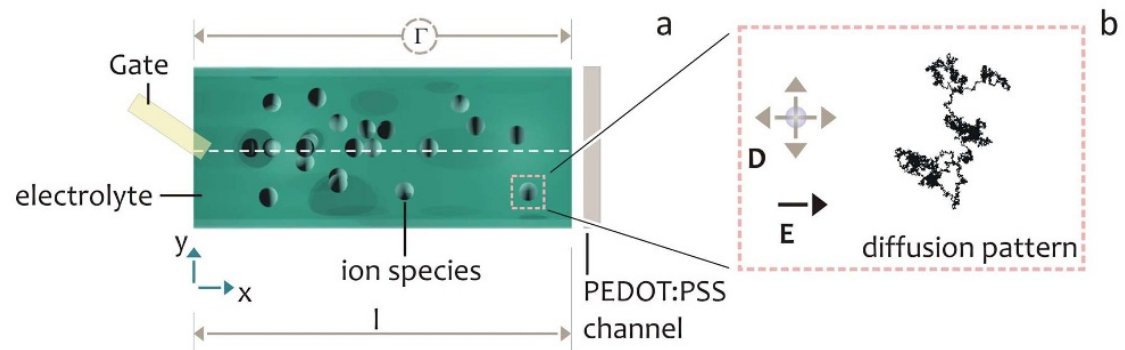

Figure $1 \mid$ Schematic representation of the ionized species within the electrolyte that, upon the application of an external voltage, are driven towards the conductive polymeric PEDOT:PSS film. The motion of the ions depend on pure drift(that is, the constant electric field) and pure diffusion. 

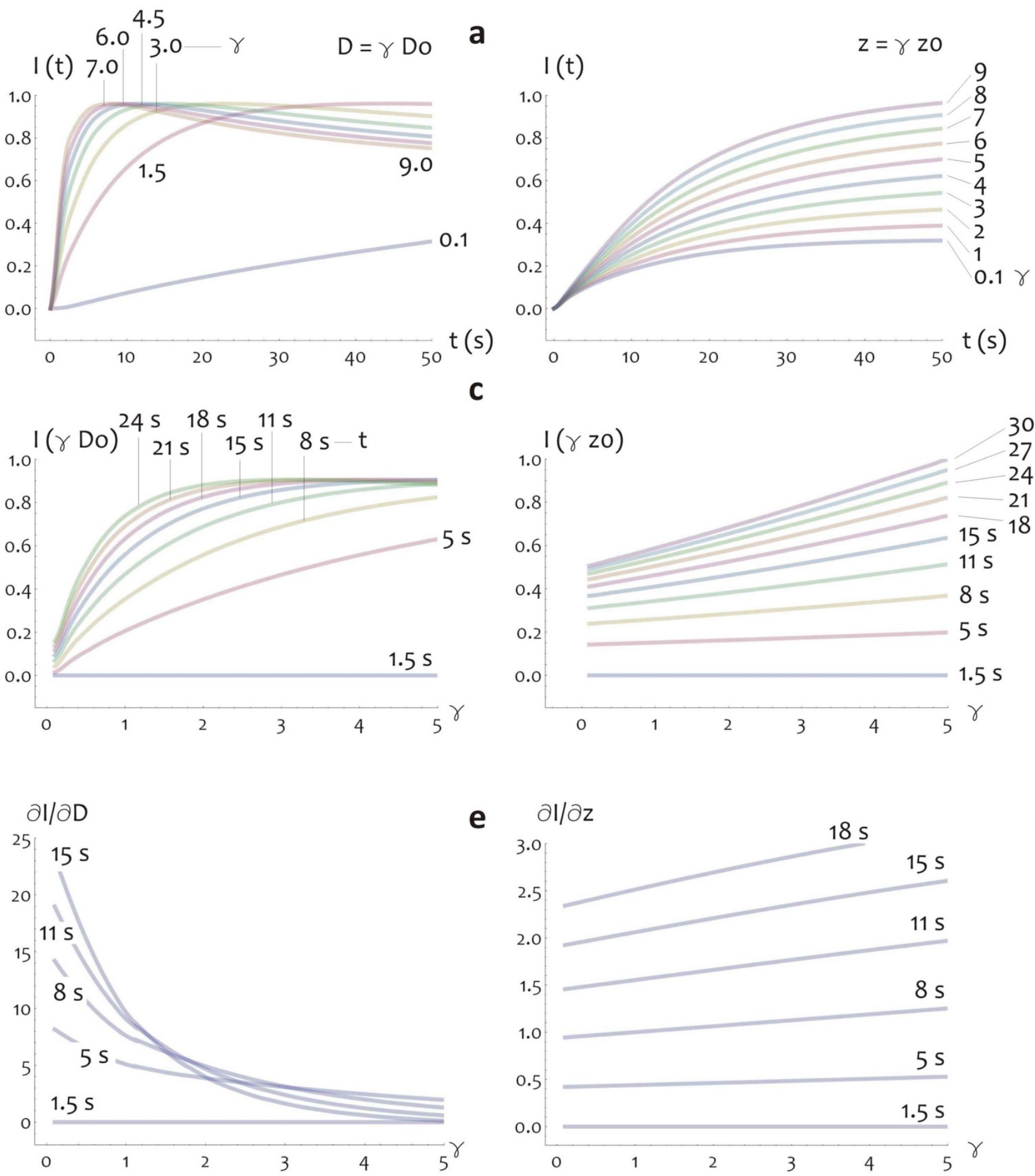

Figure 2 $\mid$ The simulated transient current $I(t)$ as a function of time for the effective diffusion coefficient varying from $10^{-1}$ to 10 times $D_{o}$, where $D_{o}=10^{-8} \mathrm{~m}^{2} / \mathrm{s}$ is an intermediate value of diffusivity used as a reference (a). The simulated transient current $\mathrm{I}(\mathrm{t})$ as a function of time for the charge number $\mathrm{z}$ varying from 0.1 to 10 (b). The simulated current as a function of $\gamma=\mathrm{D} / \mathrm{D}_{\mathrm{o}}$ for defined values of time (c).The derivative $\partial \mathrm{I} / \partial \gamma$, that is, the sensitivity analysis of I to D (e). I is reported as an explicit function of $\mathrm{z}$ at different times (d). The analysis of sensitivity of I to $\mathrm{z}$ (f).

to the other described case (effect on time constant), the rise time of the current is dominated by diffusion for small values of $\mathrm{D}$. The influence of $\mathrm{z}$ emerges for $\mathrm{D}$ larger than about $3 \mathrm{D}_{\mathrm{o}}$. Interestingly, the values of $\mathrm{k}$ range from $\sim 0.04$ to $\sim 0.4$ for $\mathrm{D}$ varying from 0.5 to about 9 times $\mathrm{D}$, and $\mathrm{z}$ from 1 to 4 . That is to say that a tenfold increment in $\mathrm{k}$ is obtained for a combined increment of $\mathrm{D}$ and $\mathrm{z}$ of nearly two orders of magnitude.
Figures $3 \mathrm{a}$ and $3 \mathrm{~b}$ are very similar to the concept of design maps for OECT sensors. Somewhat similar in concept to the periodic table of chemical elements, but more akin to phase diagrams in their graphic presentation, these design maps may provide guidance for the development of optimized OECT-biosensors and are for the time being limited to the combination of diffusion and charge. These maps and their more sophisticated evolution that will be developed 

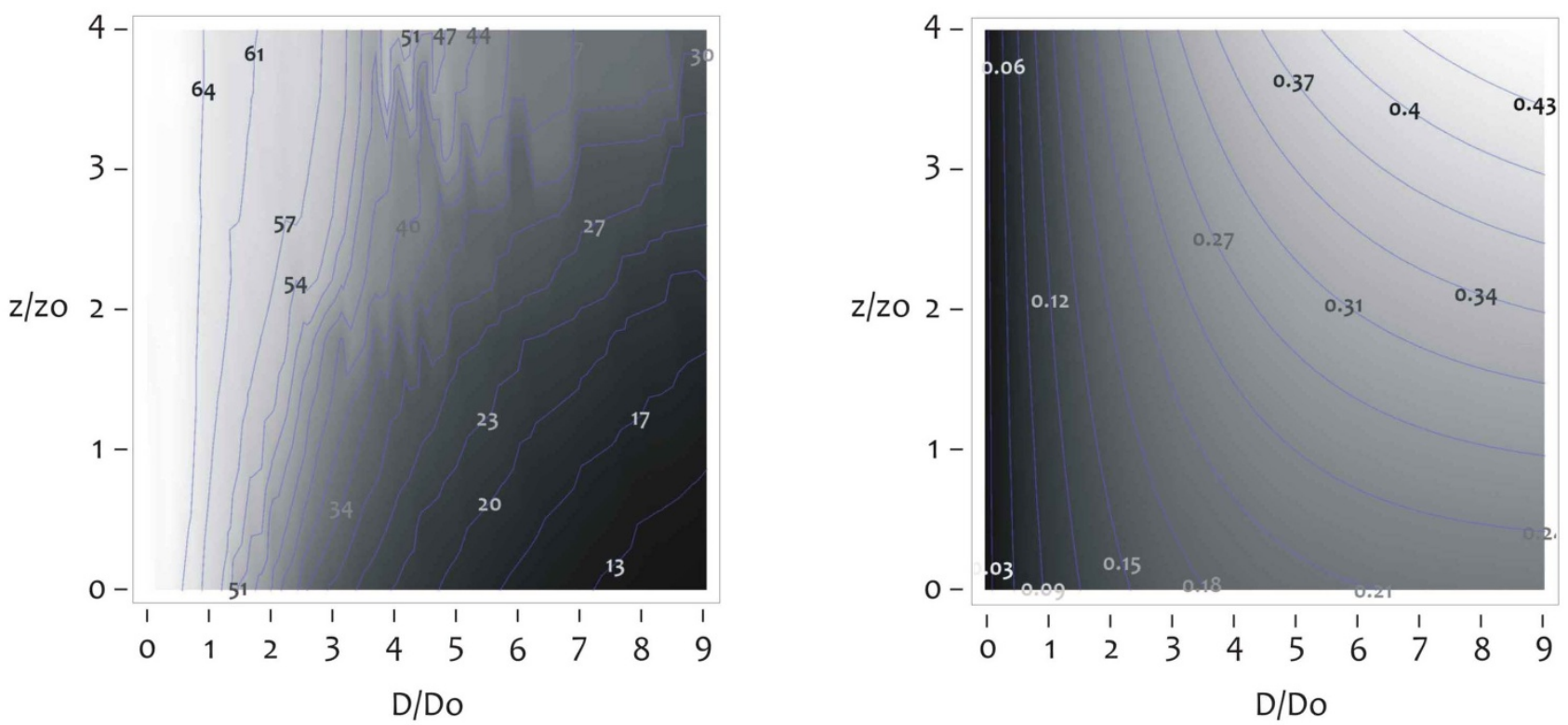

Figure $3 \mid$ The current settling time (a) and the rising time (b) of the simulated OECT sensor in the form of a contour plot as a function of concerted action of diffusion $\left(D / D_{o}\right)$ and charge $\left(z / z_{o}\right)$.

over time will allow for detection regimens to be personalized to individual cases, resulting in greater efficacy and sensitivity of the measurement and analytes detection.

Determining the diffusion coefficient of unknown species in a solution. The described model is predictive in nature as demonstrated in the above described results. More importantly, it may be realistically used to determine the physical characteristics of the transported species in a solution. This can be realized on inverting the model on experimental data. To demonstrate this, four different ions were considered in a real OECT device as described in the Materials and Methods, that are, Magnesium $\left(\mathrm{Mg}^{++}\right)$, Zinc $\left(\mathrm{Zn}^{++}\right)$, Potassium $\left(\mathrm{K}^{+}\right)$, Sodium $\left(\mathrm{Na}^{+}\right)$. These elements feature different diffusion coefficients, ranging from $\mathrm{D}=$ $0.70610^{-9} \mathrm{~m}^{2} / \mathrm{s}$ and $\mathrm{D}=0.70310^{-9} \mathrm{~m}^{2} / \mathrm{s}$ for Magnesium and Zinc, to $\mathrm{D}=1.33410^{-9} \mathrm{~m}^{2} / \mathrm{s}$ and $\mathrm{D}=1.96010^{-9} \mathrm{~m}^{2} / \mathrm{s}$, for Sodium and Potassium ${ }^{20,21}$. Moreover, the charge number of those diffusion species is also different, being $\mathrm{z}=1$ for sodium and potassium, and $\mathrm{z}=2$ for magnesium and zinc. Therefore, one would expect a different time dependent behavior of the device on changing the probed species. This is indeed the case for the measured ionic current. In Figure 4 the experimental current sensed by the device is reported as a function of time for the considered species in solution. The transient behavior for $\mathrm{Zn}$ and $\mathrm{Mg}$ is similar in shape and this is easily accepted considering that Zinc and Magnesium have physical constants that are comparable. In the same diagram,

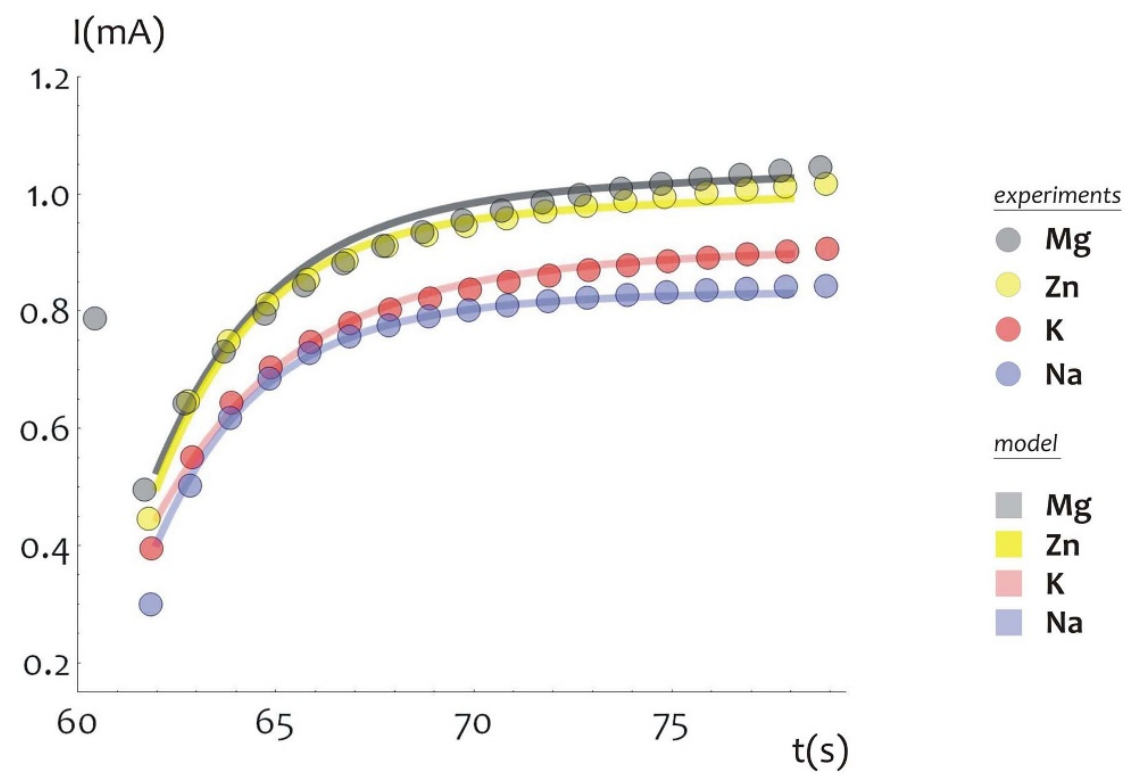

Figure 4 Experimental current sensed by the OECT device reported as a function of time for four different species in solution: $\mathrm{Mg}$, $\mathrm{Zn}, \mathrm{Na}$, $\mathrm{K}$. The experimental data were fitted using the described diffusion model; from this fitting procedure, the numerical values for the diffusion coefficients of the released species were estimated with good accuracy. 

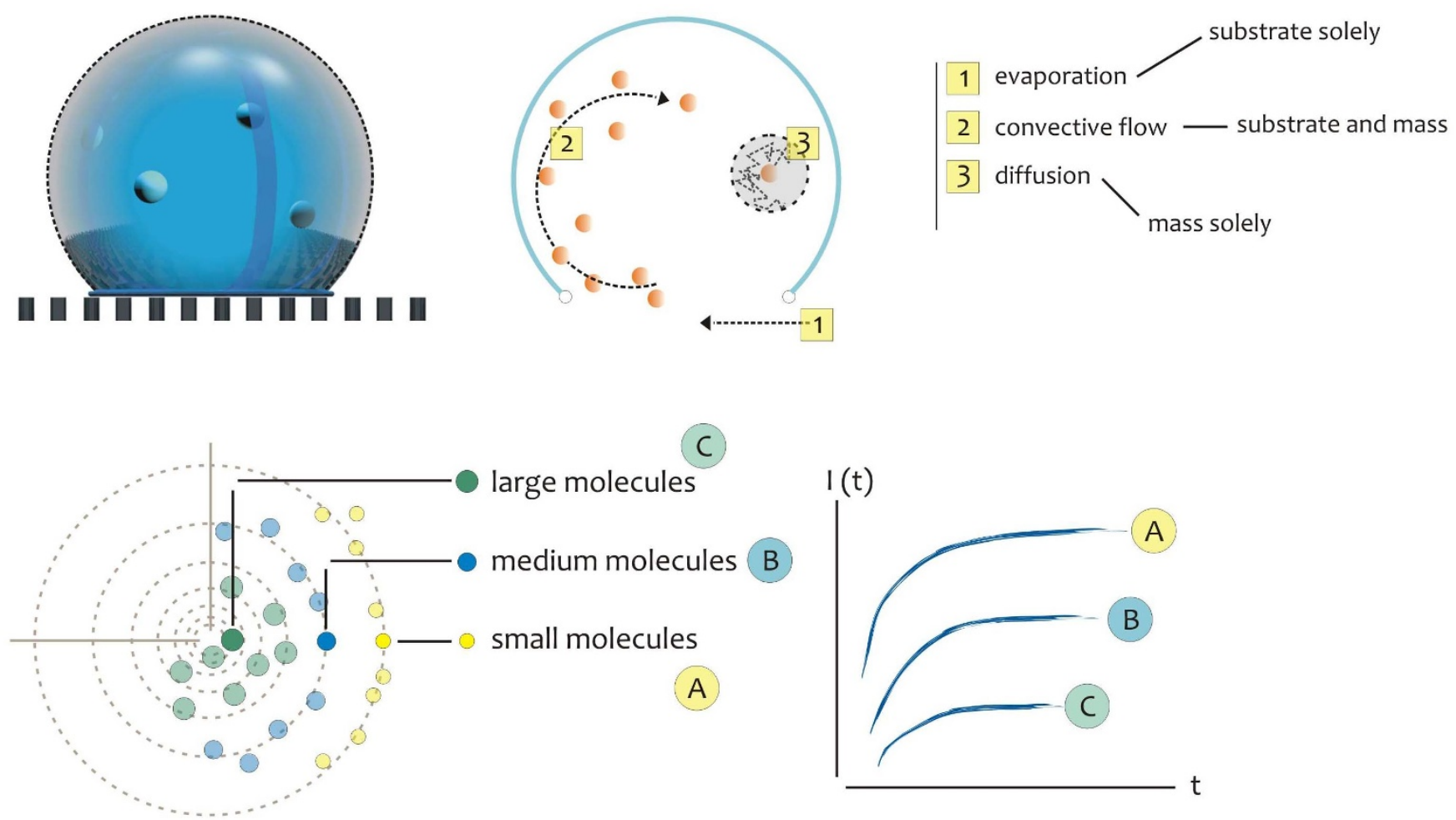

Figure $5 \mid$ On patterning the PEDOT polymeric film at the nano scale, an increasing degree of complexity is introduced into the geometry of the devices. This extra not-continuous scale would provide the device with additional functions, including the capability of manipulating extremely small volumes of a solution. For this configuration, the motion of charged species in the electrolyte is determined from the interplay of convection, diffusion, and evaporation.

differently from the case of $\mathrm{Zn}$ and $\mathrm{Mg}$, and with a lower intensity, the ionic current of Potassium (in light red) and Sodium (in light blue) are reported. Notice that $\mathrm{Mg}, \mathrm{Zn}, \mathrm{K}$ and $\mathrm{Na}$ are ordered for decreasing intensity in the diagram in Figure 4, and this is consistent with the heretofore derived results. These sets of data were therefore fitted using our described diffusion model, from this fitting procedure, the numerical values for the diffusion coefficients of the released species were estimated as $D_{M g}=0.68510^{-9} \mathrm{~m}^{2} / \mathrm{s}, D_{\mathrm{Zn}}=0.76310^{-9} \mathrm{~m}^{2} / \mathrm{s}$, $\mathrm{D}_{\mathrm{K}}=2.1010^{-9} \mathrm{~m}^{2} / \mathrm{s}, \mathrm{D}_{\mathrm{Na}}=1.21310^{-9} \mathrm{~m}^{2} / \mathrm{s}$, that are similar to the true values of diffusivity, with errors, with respect to these, of $\mathrm{e}_{\mathrm{Mg}}=$ $0.021, \mathrm{e}_{\mathrm{Zn}}=0,085, \mathrm{e}_{\mathrm{K}}=0.07, \mathrm{e}_{\mathrm{Na}}=0.09$, that are small. This would demonstrate the ability of the model to infer the characteristics of a specific specie in a solution from a simple ion current measurement. Moreover, it demonstrates that the method may be utilized to differentiate different species in a complex mixture.

\section{Discussion}

The described results demonstrate that, in considering OECT sensors, the effect of diffusion and charge of the probed species in the electrolyte cannot be neglected. In similar devices, the measurement of the ionic current, that is, the response of the system, results from a sequence of tightly interweaving processes, that are, (i) the transport of the species from the electrolyte into the conductive polymer, (ii) the injection of a cation into the organic film and the removal of a hole at the source electrode, (iii) the transport of holes between source and drain. Our results indicate that the onset of this chain of events, that is, (i) the transport of the species in the electrolyte, determines the final response of the system. The mechanisms of ion transportation in the electrolyte would depend, in turn, on the externally applied voltage, and on the electrical mobility of those ions in the solution, that is described by the well known Einstein-Smoluchowski relation ${ }^{22} \mathrm{~m}=$ $\mathrm{q} / \mathrm{KT}$ D. For a fixed temperature, the combined effect of the charge $\mathrm{q}$ and the diffusion $\mathrm{D}$ would therefore have an effective impact on the output of the sensor. Considering that the Brownian coefficient of diffusion is inversely related to the hydrodynamic diameter of a diffusing molecule, different species in a solution, with different sizes and thus mobilities, would behave differently upon the application of the same stimulus. The described mathematical method delivers the ability to predict and anticipate those differences. This understanding may have some very practical applications in bio-sensing and molecular diagnostic. In the results, we demonstrated how this scheme can be utilized for detecting and recognizing specific species in a solution. More importantly than this, we demonstrated how, using a simple fitting procedure, one may retrieve the diffusion coefficients (and thus the atomic radii/masses) of unknown species in a solution, that is to say, the method is capable to perform the label free analysis of the analytical content of a solution on a quantitative basis. Its more sophisticated evolution that will be developed over time will allow the detection of single species from a complex mixture.

However, this concept has a certain relevance beyond these specific results. Consider, for ease of visualization, the scheme in Figure 5. The theoretical model indicates that the displacement of the active ions in the electrolyte would ultimately determine the response of the system. For the present configuration, this displacement is driven by the externally applied electric fieldand by pure diffusion. It may be convenient, for specific purposes, introducing a supplementary parameter in the system i.e. the velocity vector field in the solution domain, an additional convective term. This can be done utilizing two different approaches. The first, utilizes active micro- and nano-fluidics, where active components as micro-pumps or micro valves manipulate extremely small volumes of fluids in constrained geometries at the micro and nano scale. This approach shall not be discussed here. The second (passive) approach, is based on an accurate texturing of the interface between the electrolyte and the organic polymeric film. On patterning the PEDOT polymeric film at the nano scale, an increasing degree of complexity is 
introduced into the geometry of the devices. This extra not-continuous scale would provide the device with additional functions, including the capability of manipulating extremely small volumes of a solution. Depending on texture and chemical modification, a millimetric drop would maintain a spherical shape if positioned upon those surfaces. This concept is at the base of super-hydrophobic surfaces, that are bio-mimetic, structured micro and nano-materials which reveal superior properties compared to conventional flat surfaces, including extremely low friction coefficients ${ }^{23,24}$.

An aspect in regards to which a drop is different from the same volume of liquid constrained in a rectangular geometry, is that the drop develops convective flows within its volume due to the celebrated Marangoni effect ${ }^{25}$. These flows of masses depend on the size of the dropand on its curvature, that is, on the angle of contact on the surface. Consequently, they may be predicted/calculated by controlling the geometry of the substrate. Nanotechnologies provide a tool to fabricate the substrate on the basis of a specific design to obtain a convective flux with the desired characteristics. These flows represent the additional convective term that may couple to diffusion to yield a complex field of motion that would ultimately modify the transportation of molecules from the electrolyte to the electrode, and thus the output of the measurement system. Where proven inadequate, pure analytical models can be paired to computational fluid dynamics (cfd) methods and computer simulations to resolve the field of motion in complex geometries.

Therefore, this scheme can be utilized to realize a system where diffusion and convection are separately controlled and, from the balance of these, different molecules in a mixture can be fragmentized, separated or selected. A correct design of the pattern can render this selection highly precise and reproducible. In doing so, a new generation of devices will be obtained with high sensitivity, selectivity and reliability. This concept has been exploited for some exploratory experiments reported $\mathrm{in}^{26}$, where a PEDOT conductive polymer and super-hydrophobic surfaces are, for the first time, merged together. For guaranteeing an efficient analysis, the fabrication of such devices should be always accompanied by suitable mathematical models, like that introduced in this or other similar works ${ }^{27,28}$. The use of PEDOT has heretofore been limited to direct applications, if combined with nano-geometry, it could instead represent the infrastructureof a new devices which feature new, unprecedented functions.

\section{Methods}

Following ${ }^{16}$, the behavior of the OECT device is here analyzed on dividing the system into an electronic and an ionic circuit. The electronic circuit consists of ap-type organic semiconductor film that transports holes between source and drain electrodes and is described by Ohm's law. Differently from this, the ionic circuit accounts for transport of ionic charge in the electrolyte. The interaction of these two components, namely the transport of ions from the electrolyte into the organic semiconductor film, is fundamental to the behavior of OECTs. In a separated experimental section, we shall describe the experimental set up utilized to derive the data that confirmed the feasibility of the model.

Transporting charged species into the channel. In what follows, we will describe the ionic transport of electroactive species upon the application of an external voltage. In doing this, consider the scheme in the Supporting Information Fig. S1. Assume that, at the initial time $t=0, n$ charges are uniformly distributed in a rectangular channel. Upon the application of an external voltage $\Gamma$, those charged particles will be transported across the channel. The driving forces responsible for this motion are pure diffusion and the electromagnetic force field. For this configuration, the distribution of charged particles can be described by the celebrated Nernst-Planck equation ${ }^{17-19}$

$$
\boldsymbol{J}=-D\left(\nabla \psi+\frac{z F}{R T} \psi \nabla \varphi\right)
$$

that, for mono dimensional geometries described by the sole spatial variable $\mathrm{x}$, takes the form

$$
\mathrm{J}_{\mathrm{x}}=-D\left(\frac{\partial \psi}{\partial \mathrm{x}}+\frac{z F}{R T} \psi \frac{\partial \varphi}{\partial \mathrm{x}}\right)
$$

in (2), $\mathrm{J}_{\mathrm{x}}$ is the molar flux, D is the Brownian or molecular diffusion coefficient, $\psi$ is the concentration, defined as the number of moles of charged particles per unit area, $\mathrm{z}$ is the charge number, $\mathrm{F}=9.6410^{4} \mathrm{C} \mathrm{mol}^{-1}$ is the Faraday constant, $\mathrm{R}=8.314 \mathrm{~J}$
$\mathrm{K}^{-1} \mathrm{~mol}^{-1}$ the universal gas constant, T, the absolute temperature of the system; $\varphi$, instead, is the electric potential. We make now the assumption that $\varphi$ is linear along the channel, being $\partial \varphi / \partial \mathrm{x}=\Gamma=-\mathrm{E}_{\mathrm{x}}$, where $\mathrm{E}_{\mathrm{x}}$ is longitudinal component of the electric field, and thus (2) may be rephrased as

$$
\mathrm{J}_{\mathrm{x}}=-D\left(\frac{\partial \psi}{\partial \mathrm{x}}-\frac{z F}{R T} \psi \mathrm{E}_{\mathrm{x}}\right)
$$

using in (3) the continuity condition that guarantees the conservation of mass

$$
\frac{\partial \psi}{\partial \mathrm{t}}=-\frac{\partial \mathrm{J}_{\mathrm{x}}}{\partial_{\mathrm{x}}}
$$

one would obtain the following governing equation

$$
\frac{\partial \psi}{\partial \mathrm{t}}=D\left(\frac{\partial^{2} \psi}{\partial \mathrm{x}^{2}}-\frac{\mathrm{zF}}{\mathrm{RT}} \mathrm{E}_{\mathrm{x}} \frac{\partial \psi}{\partial_{\mathrm{x}}}\right)
$$

or, that is equivalent

$$
\frac{\partial \psi}{\partial \mathrm{t}}=D \frac{\partial^{2} \psi}{\partial \mathrm{x}^{2}}-v \frac{\partial \psi}{\partial \mathrm{x}}
$$

and, on solving Eq.(6), the distribution $\Psi(\mathrm{x}, \mathrm{t})$ of the charged particles as a function of the spatial and time variables, would be determined. Eq.(6) has the form of a one dimension convection-diffusion solute transport equation, where the convective term $v$ $=\mathrm{D} \mathrm{E}_{\mathrm{x}} \mathrm{zF} / \mathrm{RT}$ is maintained constant for the extension of the channel. For the present configuration, the initial and boundary conditions are imposed as follows

$$
\begin{array}{lc}
\psi(x, 0)=\psi_{0} & 0<x<l \\
\psi(\infty, t)=0 & t>0
\end{array}
$$

Equation (6), along with the initial and boundary conditions (7), has an analytical solution that reads as ${ }^{29}$

$$
\begin{aligned}
& \psi(x, t)=\psi_{o}(\Omega(x, t)+\Upsilon(x, t)) \\
& \Omega(x, t)=\frac{1}{2} \operatorname{erf}\left(\frac{x-l-v t}{2 \sqrt{D t}}\right)+\frac{1}{2} e^{v x / D} \operatorname{erf}\left(\frac{x+l+v t}{2 \sqrt{D t}}\right) \\
& \Upsilon(x, t)=\frac{1}{2} \operatorname{erf}\left(\frac{x-v t}{2 \sqrt{D t}}\right)+\frac{1}{2} e^{v x / D} \operatorname{erf}\left(\frac{x+v t}{2 \sqrt{D t}}\right)
\end{aligned}
$$

where $l$ is the channel length, and erf is the error function, defined as

$$
\operatorname{erf}(t)=\frac{2}{\sqrt{\pi}} \int_{0}^{t} e^{-\tau^{2}} d \tau
$$

the concentration in Equation (8) indicates how, for a fixed longitudinal distance $\mathrm{x}$, the number of ions per unit area varies as a function of time. The total amount of charge that is transported into the OECT, that is, the PEDOT channel, may be accordingly derived as

$$
Q(t)=N_{A} q S \int_{0}^{t}\left(v \psi(l, \tau)-\left.D \frac{\partial \psi}{\partial x}\right|_{x=l}\right) d \tau
$$

and $\mathrm{Q}(\mathrm{t})$ is again a function of time. In $(10), \mathrm{N}_{\mathrm{A}}=6.0210^{23}$ is the Avogrado's number, $\mathrm{q}$ is the elementary charge, $\mathrm{S}$ is the cross sectional area of the Electrolyte. $\mathrm{Q}(\mathrm{t})$ would explicitly depend on $\mathrm{D}$.

Transient behavior of the drain current. The transient behavior of OECTs was solved in ${ }^{16}$.The problem was considered as the superposition of effects, that are, injection of a cation from the electrolyte into the organic film and removal of a hole at the source electrode. On neglecting the spatial variation of voltage and hole density, an expression for transient source-drain current $\mathrm{I}(\mathrm{t})$ was derived, being

$$
I(t)=G\left(1-\frac{Q(t)}{q p_{o} V}\right) V_{d}-f \frac{\partial Q(t)}{\partial t}
$$

where $\mathrm{G}=\mathrm{q} \mu \mathrm{p}_{\mathrm{o}} \mathrm{V}$ is the conductance of the organic semiconductor film, $\mu$ is the hole mobility, $\mathrm{p}_{\mathrm{o}}$ is the initial hole density, $\mathrm{V}$ is the volume of the semiconductor material, $V_{d}$ is the constant drain voltage, $f$ is a corrective constant that accounts for the non uniform distribution of the hole density. Using in (11) Eq.(10), the transient current can be accordingly determined. Similarly to the total charge $\mathrm{Q}(\mathrm{t}), \mathrm{I}(\mathrm{t})$ depends on the molecular diffusion coefficient $D$. This is shown in Fig. $S 2$, where $I(t)$ is reported as a function of time for different values of diffusivity.

Measuring the electric characteristic of ionized species in solution. Figure S3, in the Supporting Information, shows a sketch of the OECT realized on cotton fiber and of its electrical circuit. Figure $\mathrm{S} 3$ reports also a schematic representation of the mechanism underling the OECT sensing process. The saline species $(\mathrm{NaCl}, \mathrm{KCl}$, $\mathrm{MgCl}$ and $\mathrm{ZnCl}$ ) disgregate in $\mathrm{Cl}^{-}$and $\mathrm{M}^{+}$in water. In presence of a gate voltage, $\mathrm{Cl}^{-}$ ions reacts at the $\mathrm{Ag}$ electrode by an oxi-reduction process, while $\mathrm{M}^{+}$ions are forced to PEDOT:PSS channel. Hence a faradaic current, flowing in the source-gate circuit, is generated $^{5}$. Under this OECT faradaic regime of operation, the potential drop at the electrolyte/gate interface decreases and the effective gate voltage $\left(\mathrm{V}_{\text {gseff }}\right)$ increases, the 
latter forcing $\mathrm{H}^{+}$cations to move toward the polymer surface and dedope PEDOT:PSS.

Real time sensing of OECT were acquired by measuring Ids vs. time, fixing $\mathrm{Vds}$ at $-0.2 \mathrm{~V}$ and switching on $\mathrm{Vgs}$ from $0 \mathrm{~V}$ to $0.8 \mathrm{~V}$ at a fixed time, maintaining $0.8 \mathrm{~V}$ of gate potential for $60 \mathrm{~s}$. The measurements have been performed for the different salts $(\mathrm{NaCl}, \mathrm{KCl}, \mathrm{MgCl}$ and $\mathrm{ZnCl})$ with different masses and diffusion coefficient, with a concentration of $10^{-3} \mathrm{M}$. The application of $\mathrm{Vds}$ induces a drift of the holes along the PEDOT:PSS channel, generating a drain-source current (Ids). Upon application of a positive gate voltage (Vgs), cations $\left(\mathrm{M}^{+}\right)$from the electrolyte enter the PEDOT:PSS channel causing its de-doping according to the following equation ${ }^{4}$ :

$$
\text { PEDOT }^{+}: \text {PSS }^{-}+M^{+}+e^{-}=\text {PEDOT }+M^{+}: \text {PSS }^{-}
$$

The process has referred as de-doping because the cations causes a decrease of the module of drain current $|\mathrm{Ids}|$. This is due to the smaller number of holes available for conduction in PEDOT:PSS channel, as a consequence of cations incorporation into the PEDOT:PSS backbone. In fact, according to the Equation 12, cations adsorbed in PEDOT+:PSS - cause a reduction of the oxidized PEDOT + and induces a decrease in conductivity upon reduction to PEDOT0. This process results reversible and when Vgs is switched off (Vgs $=0 \mathrm{~V}$ ), ion diffusion occurs from the PEDOT:PSS to the electrolyte, increasing the number of conducting holes and, consequently, $|\mathrm{Ids}|$ (doping $)^{30}$.

1. Shirakawa, H., Louis, E., MacDiarmid, A. G., Chiang, C. K. \& Heeger, A. J. Synthesis of electrically conducting organic polymers: halogen derivatives of polyacetylene, $(\mathrm{CH}) \mathrm{x}$. Journal of the Chemical Society, Chemical Communications 578-580 (1977)

2. Dimitrakopoulos, C. D. \& Malenfant, P. R. L. Organic Thin Film Transistors for Large Area Electronics. Advanced Materials 14, 99-117 (2002).

3. Owens, R. M. \& Malliaras, G. G. Organic Electronics at the Interface with Biology. MRS Bulletin 35, 449-456 (2010).

4. Nilsson, D., Robinson, N., Berggren, M. \& Forchheimer, R. Electrochemical Logic Circuits. Advanced Materials 17, 353-358 (2005).

5. Tarabella, G., Santato, C., Yang, S. Y., Iannotta, S., Malliaras, G. G. \& Cicoira, F. Effect of the gate electrode on the response of organic electrochemical transistors. Applied Physics Letters 97, 123304 (2010).

6. Khodagholy, D. et al. High transconductance organic electrochemical transistors. Nature Communications 4, 1-6 (2013).

7. Tarabella, G. et al. Liposome sensing and monitoring by organic electrochemical transistors integrated in microfluidics. Biochimica et Biophysica Acta (BBA) General Subjects 1830, 4374-4380 (2013).

8. Tybrandt, K., Forchheimer, R. \& Berggren, M. Logic gates based on ion transistors. Nature Communications 3, 871 (2012).

9. Malliaras, G. G. Organic bioelectronics: a new era for organic electronics. Biochimica et Biophysica Acta (BBA) - General Subjects 1830, 4286-4287 (2012).

10. Bolin, M. H. et al. Active Control of Epithelial Cell-Density Gradients Grown Along the Channel of an Organic Electrochemical Transistor. Advanced Materials 21, 4379-4382 (2009).

11. Lin, P., Yan, F., Yu, J., Chan, H. L. \& Yang, M. the Application of Organic Electrochemical Transistors in Cell-Based Biosensors. Advanced Materials 22, 3655-3660 (2010)

12. Tarabella, G. et al. A single cotton fiber organic electrochemical transistor for liquid electrolyte saline sensing. Journal of Materials Chemistry 22, 23830-23834 (2012).

13. Lin, P., Luo, X., Hsing, I.-M. \& Yan, F. Organic Electrochemical Transistors Integrated in Flexible Microfluidic Systems and Used for Label-Free DNA Sensing. Advanced Materials 23, 4035-4040 (2011).

14. Yang, S. Y. et al. Electrochemical transistors with ionic liquids for enzymatic sensing. Chemical Communications 46, 7972-7974 (2010).

15. Guinovart, T., Parrilla, M., Crespo, G. A., Rius, F. X. \& Andrade, F. J. Potentiometric sensors using cotton yarns, carbon nanotubes and polymeric membranes. Analyst 138, 5208-5215 (2013).

16. Bernards, D. A. \& Malliaras, G. G. Steady-State and Transient Behavior of Organic Electrochemical Transistors. Advanced Functional Materials 17, 3538-3544 (2007).
17. Cervera, J., Ramirez, P., Manzanares, J. A. \& Mafè, S. Incorporating ionic size in the transport equations for charged nanopores. Microfluid Nanofluid 9, 41-53 (2010)

18. Corry, B., Kuyucak, S. \& Chung, S.-H. Tests of Continuum Theories as Models of Ion Channels. II. Poisson-Nernst-Planck Theory versus Brownian Dynamics. Biophysical Journal 78, 2364-2381 (2000).

19. MacGillivray, A. D. Asymptotic Solutions of the Time Dependent Nernst-Planck Equations. the Journal of Chemical Physics 52, 3126 (1970).

20. Flury, M. \& Gimmi, T. Physical Methods. Solute Diffusion. In: Methods of Soil Analysis (eds Dane, J. \& Topp, G.) (2002).

21. Samson, E. \& Marchand, J. Calculation of ionic diffusion coefficients on the basis of migration test results. Materials and Structures 36, 156-165 (2003).

22. Tammet, H. Size and mobility of nanometer particles, clusters and ions. Journal of Aerosol Science 26, 459-475 (1995).

23. Gentile, F. et al. Superhydrophobic surfaces as smart platforms for the analysis of diluted biological solutions. ACS Applied Materials and Interfaces 4, 3213-3224 (2012).

24. Gentile, F. et al. Ultra low concentrated molecular detection using super hydrophobic surface based biophotonic devices. Microelectronic Engineering 87, 798-801 (2010)

25. Deegan, R. D. et al. Capillary flow as the cause of ring stains from dried liquid drops. Nature 389, 827-829 (1997).

26. Gentile, F. et al. Microtexturing of the Conductive PEDOT:PSS Polymer for Superhydrophobic Organic Electrochemical Transistors. BioMedResearch International, DOI: http://dx.doi.org/10.1155/2014/302694.

27. Gentile, F. et al. Electroless deposition dynamics of silver nanoparticles clusters: A diffusion limited aggregation (DLA) approach. Microelectronic Engineering 98, 359-362 (2012).

28. Gentile, F. et al. Fractal structure can explain the increased hydrophobicity of nanoporous silicon films. Microelectronic Engineering 88, 2537-2540 (2011).

29. van Genuchten, M. T. \& Alves, W. J. Analytical solutions of the one-dimensional convective-dispersive solute transport equation. (eds Riverside USSL) (1982).

30. Bernards, D. A. et al. Enzymatic sensing with organic electrochemical transistors. Journal of Materials Chemistry 18, 116-120 (2008).

\section{Acknowledgments}

This work has been partially funded from project PON cod. PON01_02782, CUP B71H11000870005; from the EU Commission, the European Social Fund and the Calabria Region (POR Calabria FSE 2007-2013), and from the Italian Minister of Health (Project $n$. GR-2010-2320665).

\section{Author contributions}

N.C. designed the experimental plan, performed the current measurements, supervised the experimental part of the work and wrote the manuscript. M.V. performed the current measurements and helped in writing the manuscript. F.G. conceived the idea, designed the experimental plan, realized the theoretical model, performed the numerical calculations and statistical analysis and wrote the manuscript. All the authors discussed the results and commented on the manuscript.

\section{Additional information}

Supplementary information accompanies this paper at http://www.nature.com/ scientificreports

Competing financial interests: The authors declare no competing financial interests.

How to cite this article: Coppedè, N., Villani, M. \& Gentile, F. Diffusion Driven Selectivity in Organic Electrochemical Transistors. Sci. Rep. 4, 4297; DOI:10.1038/srep04297 (2014).

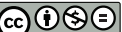

This work is licensed under a Creative Commons Attribution-

NonCommercial-NoDerivs 3.0 Unported license. To view a copy of this license, visit http://creativecommons.org/licenses/by-nc-nd/3.0 